\title{
Two new stochastic models of the failure process of a series system
}

\author{
Shaomin $\mathrm{Wu}^{1}$ \\ Kent Business School, University of Kent, \\ Canterbury, Kent CT2 7PE, United Kingdom \\ Philip Scarf ${ }^{2}$ \\ Salford Business School, Salford University, \\ Salford M5 $4 W T, U K$
}

\begin{abstract}
Consider a series system consisting of sockets into each of which a component is inserted: if a component fails, it is replaced with a new identical one immediately and system operation resumes. An interesting question is: how to model the failure process of the system as a whole when the lifetime distribution of each component is unknown? This paper attempts to answer this question by developing two new models, for the cases of a specified and an unspecified number of sockets, respectively. It introduces the concept of a virtual component, and in this sense, we suppose that the effect of repair corresponds to replacement of the most reliable component in the system. It then discusses the probabilistic properties of the models and methods for parameter estimation. Based on six datasets of artificially generated system failures and a real-world dataset, the paper compares the performance of the proposed models with four other commonly used models: the renewal process, the geometric process, Kijima's generalised renewal process, and the power law process. The results show that the proposed models outperform these comparators on the datasets, based on the Akaike information criterion.
\end{abstract}

Keywords: non-homogeneous Poisson process (NHPP), geometric process (GP), generalised renewal process (GRP), superimposed renewal process, virtual component.

$\overline{1}$ Corresponding author. Email: s.m.wu@kent.ac.uk. Telephone: 00441227827940.

2 Email: p.a.scarf@salford.ac.uk. Telephone: 00441612953817

Suggested citation: Wu, S., Scarf, P., Two new stochastic models of the failure process of a series system, European Journal of Operational Research, DOI:10.1016/j.ejor.2016.07.052 


\section{Introduction}

\subsection{Background}

Modelling the failure processes of technical systems has attracted much attention from reliability researchers. There exist many papers that attempt to develop statistical models for characterising the failure process of a system (see, Duane, 1964; Cox \& Lewis, 1966; Lam, 1988; Kijima \& Sumita, 1986; Baxter, Kijima, \& Tortorella, 1996; Dorado, Hollander, \& Sethuraman, 1997; Doyen \& Gaudoin, 2004; Wu \& Zuo, 2010; Doyen \& Gaudoin, 2011, for example). However, much of this existing research assumes that the systems are equivalent to one-component systems. Such an assumption is restrictive and unrealistic as real-world systems normally consist of very many components. In addition, in the real world, the lifetime distribution of each component may not be estimable for various reasons; for example, data on real systems often contain little or no information about the failure processes of individual components. Hence, there is a need to develop new, simple (few parameters) and elegant (richly applicable) failure process models for multi-component systems. This is the purpose of this paper.

Before we introduce our models, we define repair concepts, and two important models of imperfect repair.

\subsection{Definitions}

In reliability mathematics, the effect of maintenance upon failure of an item is typically categorised into:

- Perfect repair, in which maintenance restores the condition of a failed item to an "as good as new" status; for example, a failed item is replaced with a new identical one. The renewal process is a widely used model for the failure process of items under perfect repair (Ross, 1996).

- Minimal repair (see, Duane, 1964; Cox \& Lewis, 1966, for example), in which maintenance restores a failed item to its state immediately prior to failure. The operating state of an item after minimal repair is often called "as bad as old" in the literature. The only model of minimal repair available in the literature is the non-homogeneous Poisson process (NHPP).

- Imperfect repair, in which maintenance restores a failed item to a status somewhere between "as good as new" and "as bad as old". Many models, including the geometric process (GP) and its variants (Lam, 1988; Wang \& Pham, 1996; Wu \& Clements-Croome, 2006), the generalised renewal process models (GRP) (Kijima \& Sumita, 1986; Kijima, 1989; Doyen \& Gaudoin, 2004), and the reduction of failure hazard models (Doyen \& Gaudoin, 2004), have been developed for modelling imperfect repair. 
The particular models themselves are defined as follows.

- The geometric process: Following Lam (1988), given a sequence of non-negative random variables $\left\{X_{k}, k=1,2, \ldots\right\}$, if they are independent and the cumulative distribution function of $X_{k}$ is given by $F\left(a^{k-1} x\right)$ for $k=1,2, \ldots$, where $a$ is a positive constant, then $\left\{X_{k}, k=1,2, \cdots\right\}$ is called a geometric process (GP). The GP has attracted a lot of attention in the literature (see, Zhang, Gaudoin, \& Xie, 2015; Wu \& Scarf, 2015, for example).

- The generalised renewal process: Kijima and Sumita (1986) and Kijima (1989) introduce two types of repair models, type I and type II, using the concept of virtual age. These models distinguish between the age of the system, which is the time elapsed since the system was new (usually at time $t=0$ ), and the virtual age of the system, which accounts for the current health of the system when compared to a new system. The two models assume $V_{k}=V_{k-1}+q_{k} X_{k}$, and $V_{k}=q_{k}\left(V_{k-1}+X_{k}\right)$, respectively, where $V_{k}$ is the virtual age of the system immediately after the $k$ th repair, $X_{k}$ is the operating time of the system since the $k$ th repair, and $0 \leq q_{k} \leq 1$. The models are often referred to collectively as the generalised renewal process (GRP). In the type I model, if $q_{k}=0$, then the $k$ th repair is a perfect repair; if $q_{k}=1$, then the $k$ th repair is a minimal repair.

- The renewal process, the superimposed renewal process, and the homogeneous Poisson process (HPP): Consider a series system consisting of $m$ sockets into each of which there are inserted non-repairable independent components, and whenever a component fails, the system fails, and the failed component is replaced with a new identical one and system operation resumes. Then the number of failures occurring at each socket is a renewal process and the number of failures of the series system as a whole forms a superimposed renewal process (Høyland \& Rausand, 2009). In general, the superimposed renewal process is not a renewal process, unless the individual renewal processes are homogeneous Poisson processes (Drenick, 1960). When both the number of components in the system is large and the operation time of the system is large then the superimposed renewal process behaves approximately as a homogeneous Poisson process (HPP) (Høyland \& Rausand, 2009). The HPP is a counting process with constant intensity function (or rate of occurrence of failures).

- The non-homogeneous Poisson process (NHPP): This process generalizes the HPP and has a time-varying intensity function.

\subsection{Motivation for our models}

The failure process models such as the GRP (Gilardoni, Toledo, Freitas, \& Colosimo, 2015), GP (Wu \& Clements-Croome, 2006) and NHPP (Asfaw \& Lindqvist, 2015) do not distinguish the effect of maintenance upon failure of different components in the system. Thus, such models 
effectively consider the system as a one-component system. The use of these models is typically justified by the fact that in practice failure data are scarce, and so, even if one could model each component in the system individually and plan maintenance accordingly, such an approach would not be applicable.

Furthermore, appealing to the asymptotic behaviour of the superimposed renewal process as justification for the use of an HPP in an application is questionable because in practice typical systems are relatively young and failures are rare. Using an NHPP with an increasing intensity function such as the power-law process (Høyland \& Rausand, 2009) overcomes this system age issue. However, the NHPP (and HPP for that matter) supposes repair is minimal. At the other end of the spectrum, the renewal process supposes the entire system is replaced on failure so that repair is perfect.

Capturing imperfect repair with the GP or the GRP presents further difficulties. The GP implicitly assumes that the times between failures are either stochastically increasing or stochastically decreasing, which is not always true for the failure process of a series system. For example, if all the components in the series system have increasing hazard functions, then a replacement of a failed component improves the reliability of the system; on the other hand, operating times between adjacent replacements of the system are stochastically decreasing. Hence the times between failures of the system may be neither stochastically increasing nor stochastically decreasing. Furthermore, in the limit (large number components at large $t$ ) the superimposed renewal process behaves as a homogeneous Poisson process, which cannot be captured by the GP.

The GRP overcomes the issue of stochastically increasing or decreasing times between failures by allowing the repair effect parameter to vary. However, if indeed $q_{k}$ vary with $k$, then typically they must be estimated using a very limited number of failure observations. As a result, the models will be poorly estimated. If the $q_{k}$ are assumed equal for all $k$, then the GRP will not capture the fact that the effects of replacement of failed components of different types are different.

\subsection{Our proposed solution}

In summary then, the existing models of the failure process of a multi-component system are restrictive. Furthermore, limited failure data make it impossible to estimate either the lifetime distribution of each component in a system or a model for a system as a whole with many parameters. We must therefore seek simple and elegant models for a system as a whole that can be fitted using a limited number of failure observations. Our contribution develops two new classes of such models for a series system with non-identical components. 
In these models, the failure process of the system is regarded as equivalent to the failure process of a system consisting of a component, called the virtual component, in its socket and the remainder of the system, called the virtual sub-system (VSS). Upon a failure of the system, we suppose that the virtual component is replaced and the remainder of the system is either not maintained (equivalently minimally repaired) or subject to imperfect repair. In reality, one can contend that at a repair, the change in system reliability is at least as big as if the most reliable component had been replaced. Broadly speaking, replacement of the virtual component upon system failure captures this notion.

It should be noted then that in this paper, we distinguish three systems: i) the real system, that is, the reality, e.g. a manufacturing cell, a traction motor, a wind turbine, a compressor; ii) the system, that is, the mathematical model of the system, e.g. a series system with a number of non-repairable, non-identical components; and iii) the virtual system (VS), consisting of a virtual component (VC) and a virtual sub-system (VSS).

In our first model (Model I) the number of sockets in the series system is not specified. In our second (Model II) the number of sockets in the series system is $m$. The distinguishing features of the two models are discussed in detail later in the paper. The key concept, and a contribution of this paper, is the notion of the virtual component. Through this notion, our models capture not only that a repair effect is neither as good as new nor as bad as old but also that systems comprise distinct, typically non-identical components.

The paper considers the scenarios where the lifetime distribution of each component is neither known nor knowable for various reasons. For example, if the number of system failures is large but one does not know the causes of system failure (so that the different components cannot be distinguished), the lifetime distribution for each component cannot be estimated. On the other hand, if the number of failures is small, knowing the causes of system failures does not provide sufficient information to estimate the lifetime distribution for each component.

Thus, we claim that this paper is the first paper to model the repair effect in multi-component systems with a stochastic process, on the basis that the virtual age reduction models, such as the GRP, do not model a multi-component system, and the superimposed renewal process, HPP and NHPP do not model repair. It proposes elegant models for the failure process of a series system that overcome the limitations of existing models that are either restrictive (renewal process and its generalizations) or require knowledge of the failure process for individual components (superimposed renewal process).

The structure of the paper is as follows. Section 2 lists assumptions and notation required. Section 3 develops the two models of interest. Section 4 gives the likelihood functions of the two models given data on failures. Section 5 assesses the validity of the proposed models based on artificially generated datasets and a real-world dataset. We make some concluding remarks, 
including the implications of our work for applications, in the final section.

\section{Assumptions and notation}

Consider a series system with a number of statistically independent components. If a component fails, the system fails. On failure, the failed component is replaced instantaneously by an identical component, and the system is restored to operation. Time for replacement is negligible. Associate a socket with each component location in the system, in the sense of Ascher and Feingold (1984), so that the components in their sockets collectively perform the operational function of the system.

The system is new and functioning at time $t=0$.

Denote system failures occurring at successive time points by $T_{k}$, for $k=1,2, \ldots$, with $T_{1}<T_{2}<\ldots$ Let $X_{1}=T_{1}$ and $X_{k}=T_{k}-T_{k-1}$ for $k \geq 2$. Then, $X_{1}$ is the time to the first failure and $X_{k}$ is the time between the $(k-1)$ th and the $k$ th failures (for all $k \geq 2$ ). Let $t$ denote an arbitrary time since new, so that if $T_{k}<t<T_{k}+1$ then by time $t$ the system has experienced exactly $k$ failures. Further, we denote $t_{k}$ as the observation of $T_{k}$.

The hazard function of a component with lifetime $X$ is

$$
h(t)=\lim _{\Delta t \rightarrow 0} \frac{P\{t \leq X<t+\Delta t \mid t \leq X\}}{\Delta t} .
$$

Let $N(t)$ denote the number of failures of the system up to time $t$. The failure process of the system can be defined equivalently by the random processes $\left\{X_{k}\right\}_{k \geq 1}$ or $\{N(t)\}_{t \geq 0}$ and is characterised by the intensity function,

$$
\lambda(t)=\lim _{\Delta t \rightarrow 0} \frac{P\{N(t+\Delta t)-N(t) \geq 1 \mid \mathscr{H}(t)\}}{\Delta t},
$$

where $P\{N(t+\Delta t)-N(t) \geq 1 \mid \mathscr{H}(t)\}$ is the probability that the system fails within the interval $(t, t+\Delta t)$, given the history of failures up to time $t, \mathscr{H}(t)$ (Cox \& Lewis, 1966).

Note: when a counting process counts failures of a (non-repairable) component with a lifetime $X$, since this component can fail at most once, the intensity function of this counting process is the same as the hazard function (of the component). Therefore, for a (non-repairable) component the terms hazard and intensity are synonymous. However, for clarity, throughout this paper, where we are concerned with a component, we will use the term hazard function, and where we are concerned with the system, we will (necessarily) use the term intensity function. Their integrals $\int_{0}^{t} \lambda(u) d u$ and $\int_{0}^{t} h(u) d u$ are referred to as the cumulative intensity function (CIF) and the cumulative hazard function (CHF), respectively. Throughout the paper, $h(x)$ is a hazard function and $\lambda(x)$ is an intensity function. 


\section{Model development}

In this section we will develop the two new models. First, we recall two concepts, and then we derive a result about the cumulative intensity function of the system when the components in a series system are identical (Proposition 1).

Given a component with hazard function $h(t)$ and lifetime $X$, then

$$
\operatorname{Pr}\{X \leq y+x \mid X \geq y\}=\frac{F(x+y)-F(y)}{1-F(y)}=1-\exp \left(-\int_{y}^{x+y} h(u) d u\right)
$$

$\operatorname{Pr}\{X \leq y+x \mid X \geq y\}$ in Eq. (3) is the cumulative distribution function of the remaining lifetime distribution of a component that has survived for $y$ time units. It implies that the residual CHF of a component that has survived for $y$ time units is $\int_{y}^{x+y} h(u)$. Similarly, for a repairable system with intensity function $\lambda_{s}(u), \int_{x}^{t} \lambda_{s}(u) d u$ is the cumulative intensity over the interval $[x, t)$. For the sake of distinction, $\int_{x}^{t} \lambda_{s}(u) d u$ is referred to as the cumulative residual intensity function (CRIF) when $x \neq 0$. Here, with the assumption that an item is subject to minimal repair, the CIF $\int_{0}^{t} \lambda_{s}(u) d u$ is the expected number of failures of this item in $[0, t)$, whereas the CRIF $\int_{x}^{t} \lambda_{s}(u) d u$ is the expected number of failures in the time interval $(x, t)$.

Note, for a series system consisting of $m$ components, each of which has hazard function $h_{i}(t)$ (for $i=1,2, \ldots, m$ ), then the intensity function of the system prior to first failure is $\sum_{i=1}^{m} h_{i}(t)$. Proposition 1 Consider a series system with $m$ identical and statistically independent components. If a component fails, it is replaced with a new identical one immediately and system operation resumes. Let $h_{0}(t)$ denote the hazard function of a component and $H_{0}(t)=\int_{0}^{t} h_{0}(x) d x$ be the corresponding CHF. Then, for $t<t_{1}$, the CIF of the system before the first failure is $m H_{0}(t)$. Between the $k$ th and $(k+1)$ th failures of the system $(k=1,2, \ldots)$, that is, after $t_{k}$ time units, the CRIF is given by

$$
H_{0}\left(t-t_{k}\right)+\Phi_{k}(t)+\Psi_{k}(t)
$$

where

$$
\begin{aligned}
& \Phi_{k}(t)= \begin{cases}0 & \text { if } k \geq m, \\
(m-k) \int_{t_{k}}^{t} h_{0}(x) d x & \text { if } 1 \leq k \leq m-1,\end{cases} \\
& \Psi_{1}(t)=0
\end{aligned}
$$

and $\Psi_{k}(t)$ is a function of $t, t_{1}, t_{2}, \ldots$, and $t_{k}$ for $k \geq 2$

Proof. 
(a) Since a failed component is replaced with a new identical component, at time $t$ between the $k$ th and $(k+1)$ th failure of the system $\left(t_{k}<t<t_{k}+1\right)$, the CHF of the most recently installed component of the system is $\int_{0}^{t-t_{k}} h_{0}(x) d x$.

(b) When $1 \leq k \leq m-1$, at time $t_{k}<t<t_{k}+1$, in addition to the component discussed in (a), $k-1$ components have been replaced. Therefore, at least $m-k$ components have never been replaced and have been operating since $t=0$. The CRIF of the subsystem of these $m-k$ components is $(m-k) \int_{t_{k}}^{t} h_{0}(x) d x . \Psi_{k}(t)$ is the CRIF of the subsystem of the other $k-1$ components.

(c) When $k \geq m$, following the $k$ th repair the most recently replaced component has CHF $\int_{0}^{t-t_{k}} h_{0}(x) d x$. Further, the existence of a set of components that have never failed cannot be established, therefore the CRIF of the system can only be partitioned into the two terms $\int_{0}^{t-t_{k}} h_{0}(x) d x$ and $\Psi_{k}(t)$.

(d) The CRIF of the series system is then the sum of the three terms $H_{0}\left(t-t_{k}\right)+\Phi_{k}(t)+$ $\Psi_{k}(t)$. Additivity holds because components are independent so that the counting process of system failure is the sum of the counting processes of component failures.

Alternatively, one can understand the summation in Eq. (4) as: the CRIF of the most recently replaced component is $H_{0}\left(t-t_{k}\right)$ and that of a subset of the never replaced components is $\Phi_{k}(t)+\Psi_{k}(t)$.

Note, the cumulative intensity function (CIF) of the system (from time 0) can be obtained by summing the CRIF over each of intervals between failure/repair.

The idea of Proposition 1 is that the CRIF of a series system can be partitioned into a known term $m H_{0}(t)$ for the time before the first system failure, or a known term $H_{0}\left(t-t_{k}\right)+\Phi_{k}(t)$ for the time after the first system failure, and an unknown term $\Psi_{k}(t)$, where $\Phi_{k}(t)$ might be modelled with the CRIF of a convenient counting process, such as a homogeneous Poisson process (HPP) or non-homogeneous Poisson process (NHPP).

Proposition 1 inspires us to develop two models (in the following subsections) for series systems with non-identical components that are useful when data about system failures (in data collected for the system) is insufficient to estimate the lifetime distribution for each component of a system. The main idea of the two models, which we call Model I and Model II, is to represent a series system with a virtual series system. The virtual series system in Model I is composed of two virtual components (VCs): $\mathrm{VC} 1$ and $\mathrm{VC} 2$, and the virtual series system in Model II is composed of a VC and a virtual sub-system (VSS).

Remark 1 The VC1 in Model I and the VC in Model II is replaced with an identical virtual component (VC) upon failure of the system. The justification for this idea is as follows. For a series system with components with hazard functions $h_{i}(t)(i=1,2, \ldots m)$, if a component fails at $t_{k}$ and is replaced with a new identical one, then the contribution this new component 
makes at time $t_{k}<t<t_{k}+1$ to the CRIF of the system is $\int_{0}^{t-t_{k}} h_{i}(x) d x$. Furthermore, although the components that fail at time points $t_{k}(k=1,2, \ldots)$ may be different and are unknown, the component that failed at $t_{k}$ has a hazard function at least as big as $h_{c}(t)=\inf _{1 \leq i \leq m} h_{i}(t)$, and we associate this infimum hazard function $h_{c}(t)$ with the virtual component. For example, consider a series system with three components with hazard functions $0.4 t, 0.45 t$, and $0.5 t$, respectively. At $\tau$ time units following the most recent replacement, the CRIF of the system will have a contribution that is either $\int_{0}^{\tau} 0.4 u d u$ or $\int_{0}^{\tau} 0.45 u d u=\int_{0}^{\tau} 0.4 u d u+\int_{0}^{\tau} 0.05 u d u$ or $\int_{0}^{\tau} 0.5 u d u=\int_{0}^{\tau} 0.4 u d u+\int_{0}^{\tau} 0.1 u d u$, and the common contribution $\int_{0}^{\tau} 0.4 u d u$ is the cumulative hazard function of the virtual component. That is, the change in system reliability at each replacement is at least as big as if the most reliable component had been replaced.

In Model I, virtual component 2 (VC2) represents the remainder of the system, and in Model II the virtual sub-system (VSS) represents the remainder of the system, and at the $k$-th system repair VC2 and VSS are respectively not maintained and imperfectly repaired.

\subsection{Model I: when the number of components is unspecified}

Model I is a counting process with CIF and CRIF defined as follows. For $t<t_{1}$ (prior to the first failure) the CIF of the system is $\int_{0}^{t}\left(h_{\mathrm{c}}(x)+\lambda_{\mathrm{s}}(x)\right) d x$, and between the $k$ th and $(k+1)$ th failure of the system $(k=1,2, \ldots)$ the CRIF is given by

$$
\Omega_{1, k}(t)+\Omega_{2, k}(t)
$$

where

$$
\begin{aligned}
& \Omega_{1, k}(t)=\int_{0}^{t-t_{k}} h_{\mathrm{c}}(x) d x \\
& \Omega_{2, k}(t)=\int_{t_{k}}^{t} \lambda_{\mathrm{s}}(x) d x,
\end{aligned}
$$

and $h_{\mathrm{c}}(x)$ is the hazard function of VC1. The first term in (5) corresponds to VC1, which is (virtually) replaced on failure and the second term corresponds to VC2 (the remainder of the system), on which no maintenance is carried out, so that $\lambda_{\mathrm{s}}(x)$ might for example be modelled with an HPP or alternatively a NHPP with power-law intensity. This model assumes that whenever the system fails, VC1 is replaced a new identical VC. We use Figure 1 to illustrate our idea. Thus:

- if VC1 fails, a new identical VC will replace it and no maintenance is performed on VC2;

- if $\mathrm{VC} 2$ fails, it is minimally repaired and simultaneously VC1 is replaced with a new identical VC. This means that the VC1 is replaced with a new identical VC whenever it fails or VC2 fails, so that $\mathrm{VC} 1$ is renewed upon system failure, regardless of which virtual component 
failed.

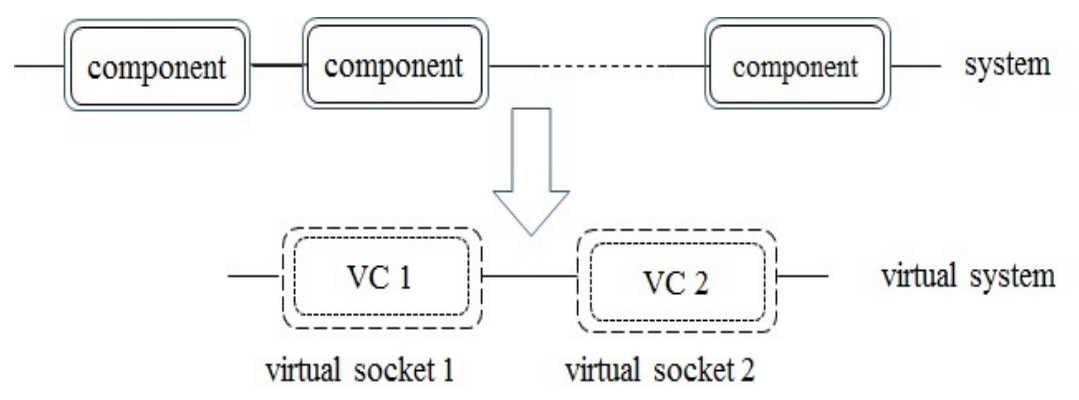

Fig. 1. The system and its corresponding virtual system in Model I.

\subsection{Model II: when the number of components is specified}

Model II is a counting process with CIF and CRIF defined as follows. For $t<t_{1}$ (prior to the first failure) the CIF of the system is $\int_{0}^{t}\left(h_{\mathrm{c}}(x)+\lambda_{\mathrm{s}}(x)\right) d x$, and between the $k$ th and $(k+1)$ th failure of the system $(k=1,2, \ldots)$ the CRIF is given by

$$
\Omega_{1, k}(t)+\Omega_{2, k}(t)
$$

where

$$
\Omega_{1, k}(t)=\int_{0}^{t-t_{k}} h_{\mathrm{c}}(x) d x
$$

and

$$
\Omega_{2, k}(t)= \begin{cases}\int_{t_{1}}^{t} \lambda_{\mathrm{s}}(x) d x & \text { if } k=1 \\ \frac{1}{m-1}\left((m-k) \int_{t_{k}}^{t} \lambda_{\mathrm{s}}(x) d x+\sum_{i=1}^{k-1} \int_{t_{k}-t_{k-i}}^{t-t_{k-i}} \lambda_{\mathrm{s}}(x) d x\right) & \text { if } 2 \leq k \leq m-1 \\ \frac{1}{m-1} \sum_{i=1}^{m-1} \int_{t_{k}-t_{k-i}}^{t-t_{k-i}} \lambda_{\mathrm{s}}(x) d x & \text { if } k \geq m\end{cases}
$$

Again, $h_{\mathrm{c}}(x)$ is the hazard function of VC1. The first term in the CRIF of the system corresponds to $\mathrm{VC} 1$, which is (virtually) replaced on failure and the second term corresponds to the virtual sub-system (VSS) (the remainder of the system).

The interpretation of Eq. (6) is that the VC in socket 1 is replaced with a new identical VC whenever it fails or the VSS fails, whichever occurs first.

On the VSS, we make the following remarks.

- For $t<t_{1}$, the intensity function of the VSS is denoted by $\lambda_{s}(t)$, and the $m-1 \mathrm{VCs}$ in the VSS are assumed to be identical and the intensity function of each VC is $\frac{1}{m-1} \int_{t_{1}}^{t} \lambda_{\mathrm{s}}(x) d x$.

- After the first failure of the system, the VC in socket 1 is renewed and its CHF becomes $\Omega_{1,1}(t)=\int_{0}^{t-t_{1}} h_{\mathrm{c}}(x) d x$; the VSS has operated $t_{1}$ time units and hence its CRIF becomes 
$\int_{t_{1}}^{t} \lambda_{\mathrm{s}}(x) d x$

- After $k$ failures $(2 \leq k \leq m-1)$ have occurred, in addition to the component that has been renewed, at most $k-1$ VCs have been replaced since time zero. This implies that at least $m-k$ VCs have not been replaced and the CRIF of the virtual subsystem consisting of the $m-k$ VCs at time $t$ is $\frac{m-k}{m-1} \int_{t_{k}}^{t} \lambda_{\mathrm{s}}(x) d x$. The exact replacement history of the other $k-1 \mathrm{VCs}$, however, is unknown, hence one may assume that $\mathrm{VC}_{i}$ was installed at time $t_{k-i}$ $(i=1,2, \ldots, k-1)$. The CRIF at time $t$ of the virtual sub-system consisting of those $k-1$ VCs is $\frac{1}{m-1} \sum_{i=1}^{k-1} \int_{t_{k}-t_{k-i}}^{t-t_{k-i}} \lambda_{\mathrm{s}}(x) d x$.

- For $k \geq m$, after $k$ failures, the CRIF at time $t$ of the $m-1$ virtual components is $\int_{t_{k}-t_{k-i}}^{t-t_{k-i}} \lambda_{\mathrm{s}}(x) d x, i=1, \ldots, m-1$.

It can be seen from the above discussion that

- On failure of the system, VC1 is replaced (renewed) and the oldest VC in the VSS is assumed to be the one replaced at the previous failure. In this way, each VC in the VSS is replaced every $m-1$ failures for $k \geq m$.

The system and virtual system are schematically illustrated in Figure 2.

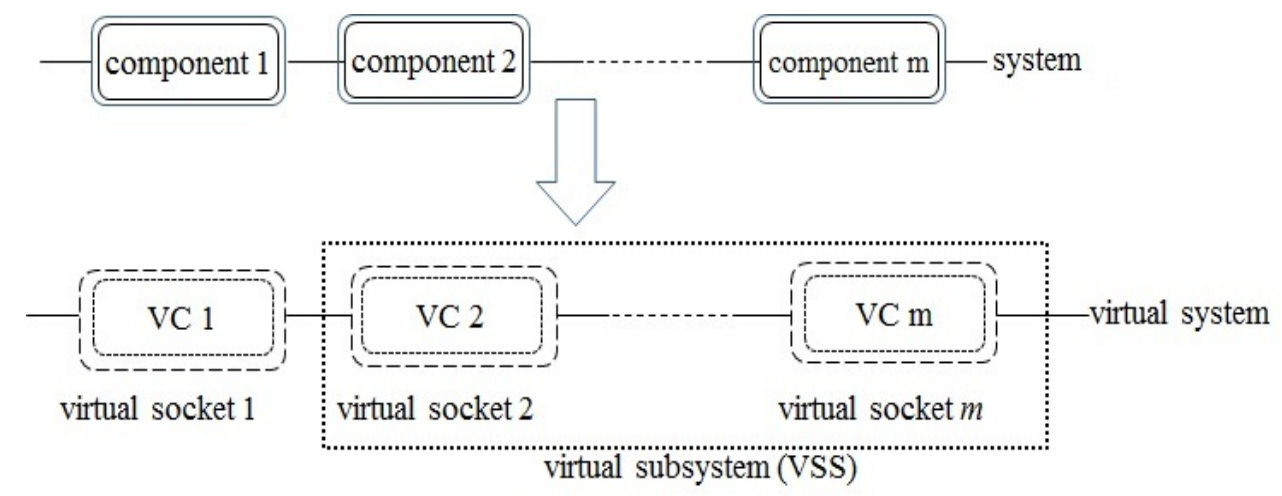

Fig. 2. The system and its corresponding virtual system in Model II.

The replacement of VCs in the VS is best illustrated by an example. In Figure 3, we show four failure times $t_{1}, t_{2}, t_{3}, t_{4}$ in a 3 component system. Then the virtual system is composed of a VC and a VSS with two VCs. The three elements in the vector are the ages of the VCs immediately after replacement on failure. The first element in the vector corresponds to VC1 in the virtual system; the second and the third elements in the vector correspond to the two VCs in the VSS.

- At time $t_{1}, \mathrm{VC} 1$ is replaced, $\mathrm{VC} 2$ and $\mathrm{VC} 3$ remain unchanged

- At time $t_{2}, \mathrm{VC} 1$ is replaced. VC2 is assumed to be replaced at time $t_{1}$, VC3 remains unchanged;

- At time $t_{3}, \mathrm{VC} 1$ is replaced. VC2 remains unchanged, VC3 is assumed to be replaced at time $t_{2} ;$ and 
- At time $t_{4}, \mathrm{VC} 1$ is replaced. VC2 is assumed to be replaced at time $t_{3}$, VC3 remains unchanged.

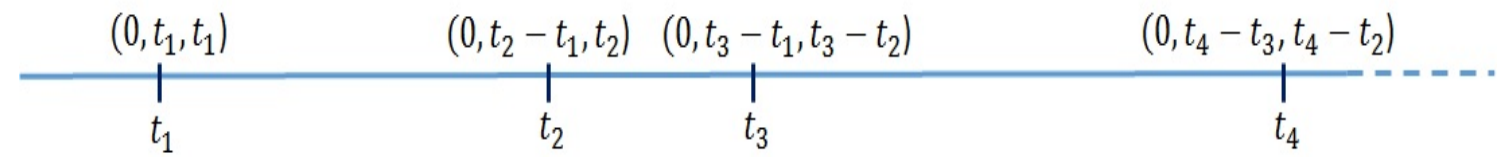

Fig. 3. Failures of the virtual system corresponding a 3-component series system in Model II, showing the ages of the VCs immediately after successive system repairs

\subsection{Some properties}

Let $\left\{X_{k}: k \geq 1\right\}$ be a sequence of nonnegative real-valued random variables with cdf $F_{k}(t)$ that have finite mean $\mathbb{E}\left[X_{k}\right]$. To avoid trivialities, we assume that $P\left(X_{k}>0\right)>0$. Let

$$
S_{n} \equiv \sum_{k=1}^{n} X_{k}, n \geq 1
$$

with $S_{0} \equiv 0$ and

$$
N(t) \equiv \max \left\{n \geq 0: S_{n} \leq t\right\}, t \geq 0 .
$$

Let $F_{k}(t)=1-e^{-\Omega_{1, k}(t)-\Omega_{2, k}(t)}$ and $X_{k}$ follow cdf $F_{k}(t)$.

Denote $\mathbb{E}\left[N_{\mathrm{c}}(t)\right]$ as the renewal function corresponding to the renewal process with the lifetime distribution $1-e^{-\int_{0}^{t} h_{c}(x) d x}$.

Lemma 1 The expected cumulative number of failures in time interval $(0, t]$ of the stochastic process defined in Model I, denoted by $\mathbb{E}\left[N_{\mathrm{I}}(t)\right]$, satisfies

$$
\max \left\{\mathbb{E}\left[N_{\mathrm{c}}(t)\right], \int_{0}^{t} \lambda_{\mathrm{s}}(x) d x\right\} \leq \mathbb{E}\left[N_{\mathrm{I}}(t)\right] \leq \mathbb{E}\left[N_{\mathrm{c}}(t)\right]+\int_{0}^{t} \lambda_{\mathrm{s}}(x) d x
$$

Proof. The system fails if either VC1 or VC2 fails. This implies the following two points.

- It is known that the reliability of a series system is smaller than the reliability of the weakest component in the system. Hence, the expected number of failures of the system up to time $t$ is greater than the expected number of failures of the weakest component up to time $t$. This proves the first inequality in Lemma (1).

- The expected number of failures of the system up to time $t$ is smaller than the sum of the expected number of failures of $\mathrm{VC} 1$ and that of $\mathrm{VC} 2$ up to time $t$. This proves the second inequality in Lemma (1).

This proves Lemma 1 
Lemma 1 holds as the VC in socket 1 in Model I is replaced with a new identical VC whenever it fails or the virtual system fails.

Lemma 2 If $\lambda_{\mathrm{s}}(x)$ is increasing in $x$, and $h_{\mathrm{c}}(x)$ and $\lambda_{\mathrm{s}}(x)$ in Model I are the same as those in Model II, respectively, then the expected cumulative number of failures by time t of the stochastic process defined in Model II, denoted by $\mathbb{E}\left[N_{\mathrm{II}}(t)\right]$, satisfies

$$
\mathbb{E}\left[N_{\mathrm{II}}(t)\right] \leq \mathbb{E}\left[N_{\mathrm{I}}(t)\right]
$$

Proof. Note that the system in Model I is composed of VC1 and VC2, and the system in Model II is composed of VC1 and the VSS. The two VC1s in the two systems have the same hazard function, which implies that the expected numbers of failures of these two VCs in $(0, t]$ are equal.

On the other hand, since $\lambda_{\mathrm{s}}(x)$ is increasing in $x$, i.e., $\int_{t_{k}-t_{k-i}}^{t-t_{k-i}} \lambda_{\mathrm{s}}(x) d x<\int_{t_{k}}^{t} \lambda_{\mathrm{s}}(x) d x$, it implies that $\frac{1}{m-1}\left((m-k) \int_{t_{k}}^{t} \lambda_{\mathrm{s}}(x) d x+\sum_{i=1}^{k-1} \int_{t_{k}-t_{k-i}}^{t-t_{k-i}} \lambda_{\mathrm{s}}(x) d x\right)<\int_{t_{k}}^{t} \lambda_{\mathrm{s}}(x) d x$. That is, $\Omega_{2, k}(t)$ in Model II is smaller than $\Omega_{2, k}(t)$ in Model I. Hence, the expected number of failures up to time $t$ of the VSS in the system associated with Model II is smaller than the expected number of failures up to time $t$ of $\mathrm{VC} 2$ in the system associated with Model I.

Considering both systems are series systems, we have $\mathbb{E}\left[N_{\mathrm{II}}(t)\right] \leq \mathbb{E}\left[N_{\mathrm{I}}(t)\right]$.

This proves Lemma 2

Lemmas 1 and 2 provide bounds on the expected number of replacements under Model I and Model II. This has practical implications for lifecycle costing, for example.

Remark 2 - Replacement occurs in socket 1 of the virtual system in both Model I and Model II whenever the system fails, even if the VC in the socket does not fail. Hence, the sequence of failures occurring at socket 1 cannot be considered a renewal process.

- Model II has three cases with different forms of CRIF: in the third case when $k \geq m$, the term $\Omega_{2, k}(t)=\frac{1}{m-1} \sum_{i=1}^{m-1} \int_{t_{k}-t_{k-i}}^{t-t_{k-i}} \lambda_{\mathrm{s}}(x) d x$ can model a state closer to equilibrium than the state in the second and first case with $C R I F=\int_{t_{1}}^{t} \lambda_{\mathrm{s}}(x) d x$ and the state with CRIF= $\frac{1}{m-1}\left((m-k) \int_{t_{k}}^{t} \lambda_{\mathrm{s}}(x) d x+\sum_{i=1}^{k-1} \int_{t_{k}-t_{k-i}}^{t-t_{k-i}} \lambda_{\mathrm{s}}(x) d x\right)$. This is a useful property of Model II, since Drenick (1960) states that the failure process of a large series system "always tends toward (statistical) equilibrium as the time of operation becomes very large", which implies that such a failure process has two states: a transient state and an equilibrium state, as interpreted by Meeker and Escobar (1998). Model I cannot model a failure process with two such states.

- It should be noted that the second term $\Omega_{2, k}(t)$ in Model II (see the quantity (6)) differs 
from the arithmetic reduction of intensity models proposed by Doyen and Gaudoin (2004). The three ARI models, which are $A R I_{\infty}, A R I_{1}$ and $A R I_{m}$, can be re-written as $\lambda(t)-$ $g\left(t_{N(t)}, t_{N(t)-1}, \ldots, t_{1}\right)$, where $g($.$) is a function of \left(t_{N(t)}, t_{N(t)-1}, \ldots, t_{1}\right)$. Their corresponding CRIFs are $\int_{t_{k}}^{t}\left(\lambda(x)-g\left(t_{N(t)}, t_{N(t)-1}, \ldots, t_{1}\right)\right) d x$, in which the upper limit of the integral is $t$, which is different from $\Omega_{2, k}(t)$. In essence, the ARI models are developed for a one-component system that is assumed to start operation at $t=0$. However, $\Omega_{2, k}(t)$ in Model II is for a multicomponent system, in which the components start operating at different time points.

Remark 3 When the number of components in Model II is not specified, one can fit Model II when assuming $m=2,3, \ldots$ and then choose $m$ so that some model selection criterion (e.g. AIC) is optimised.

\section{Parameter estimation}

Consider $M$ independent systems of the same kind (replicates), each of which consists of $m$ non-repairable components with hazard functions $h_{i}(t)$ for $i=1, \ldots, m$. Suppose that $M_{j}$ failures of system $j$ are observed at times $t_{j, 1}, t_{j, 2}, \ldots, t_{j, M_{j}}$ (where $j=1,2, \ldots, M$ ), respectively. Denote $x_{j, 1}=t_{j, 1}$ and $x_{j, k}=t_{j, k}-t_{j, k-1}$ for $k \geq 2$ and all $j$.

As discussed above, for both Model I and Model II, we suppose (a) upon system failure, VC1 in both Model I or Model II is always renewed, and (b) upon system failure, VC2 in Model I follows an NHPP. For a given system $j$, immediately after the $k$ th failure, the timeto-failure distribution is $F_{k j}(t)=1-R_{1, j k}(t) R_{2, j k}(t)$ with $R_{1, j k}(t)=\exp \left(-\int_{0}^{t-t_{j, k}} h_{\mathrm{c}}(x) d x\right)$ and $R_{2, j k}(t)=\exp \left(-\int_{t_{j, k}}^{t} \lambda_{\mathrm{s}}(x) d x\right)$. Then the likelihood function is $f_{j 0}(t) \prod_{k=1}^{M_{j}-1} f_{j k}(t)$, where $f_{j 0}(t)=\left(h_{\mathrm{c}}\left(x_{j, 1}\right)+\lambda_{\mathrm{s}}\left(x_{j, 1}\right)\right) \exp \left(-\int_{0}^{x_{j, 1}} h_{\mathrm{c}}(x) d x-\int_{0}^{x_{j, 1}} \lambda_{\mathrm{s}}(x) d x\right)$. Similarly, the likelihood function for Model II can be developed. The reader is referred to Andersen, Borgan, Gill, and Keiding (1993) for methods of statistical inference for counting processes. We then obtain the following.

Model I. The likelihood for Model I is

$$
\begin{aligned}
L_{\mathrm{I}}= & \prod_{j=1}^{M}\left\{\left(h_{\mathrm{c}}\left(x_{j, 1}\right)+\lambda_{\mathrm{s}}\left(x_{j, 1}\right)\right) \exp \left(-\int_{0}^{x_{j, 1}} h_{\mathrm{c}}(x) d x-\int_{0}^{x_{j, 1}} \lambda_{\mathrm{s}}(x) d x\right)\right. \\
& \left.\times \prod_{k=1}^{M_{j}-1}\left(h_{\mathrm{c}}\left(x_{j, k+1}\right)+\lambda_{\mathrm{s}}\left(t_{j, k+1}\right)\right) \exp \left(-\int_{0}^{x_{j, k+1}} h_{\mathrm{c}}(x) d x-\int_{t_{j, k}}^{t_{j, k+1}} \lambda_{\mathrm{s}}(x) d x\right)\right\} .
\end{aligned}
$$


Model-II. The likelihood for Model II is

$$
\begin{aligned}
L_{\mathrm{II}}=\prod_{j=1}^{M}\{ & {\left[\left(h_{\mathrm{c}}\left(x_{j, 1}\right)+\lambda_{\mathrm{s}}\left(x_{j, 1}\right)\right) \exp \left(-\int_{0}^{x_{j, 1}} h_{\mathrm{c}}(x) d x-\int_{0}^{t_{j, 1}} \lambda_{\mathrm{s}}(x) d x\right)\right] } \\
& \times\left[\left(h_{\mathrm{c}}\left(x_{j, 2}\right)+\lambda_{\mathrm{s}}\left(t_{j, 2}\right)\right) \exp \left(-\int_{0}^{x_{j, 2}} h_{\mathrm{c}}(x) d x-\int_{t_{j, 1}}^{t_{j, 2}} \lambda_{\mathrm{s}}(x) d x\right)\right] \\
& \times \prod_{k=2}^{m-1}\left[\left(h_{\mathrm{c}}\left(x_{j, k+1}\right)+\frac{1}{m-1}\left((m-k) \lambda_{\mathrm{s}}\left(t_{j, k+1}\right)+\sum_{i=1}^{k-1} \lambda_{\mathrm{s}}\left(t_{j, k+1}-t_{j, k-i}\right)\right)\right) \times\right. \\
& \times \prod_{k=m}^{M_{j}-1}\left[\left(h_{\mathrm{c}}\left(x_{j, k+1}\right)+\frac{1}{m-1} \sum_{i=1}^{t_{j, k+1}} \lambda_{\mathrm{s}}\left(t_{j, k+1}-t_{j, k-i}\right)\right) \times\right. \\
& \exp \left(-\int_{t_{j, k}}^{x_{j, k+1}} h_{\mathrm{c}}(x) d x-\frac{1}{m-1}\left(\left(m-\sum_{i=1}^{t_{j, k+1}-t_{j, k-i}} \int_{t_{j, k}-t_{j, k-i}} \lambda_{\mathrm{s}}(x) d x\right)\right)\right]
\end{aligned}
$$

One can then obtain the parameters in Model I and Model II via maximising the logarithm of $L_{\mathrm{I}}$ and the logarithm of $L_{\mathrm{II}}$, respectively.

\section{$5 \quad$ Numerical examples}

\subsection{Simulation study}

In this subsection, we fit Models I and II, and four other models (RP, NHPP, GP, and GRP) to artificially generated datasets, and then compare the AIC values of these models. It is known that the renewal process (RP) is usually used for perfect repair scenarios, the non-homogeneous Poisson process with power law intensity function (NHPP-PL) is often used for minimal repair, and two models for imperfect repair: the geometric process (GP) and the generalised renewal process with $V_{k}=V_{k-1}+q X_{k}$ being the virtual age after the $k$ th failure (GRP) are used for imperfect repair. When Model $\mathrm{I}$ is used, the number $m$ of components in the system is not specified.

The following steps are followed to compare the performance of the models.

(1) We consider three cases $m=5,10,15$ for the number of sockets in the system.

(2) The time to failure of each component follows a Weibull distribution $1-e^{-\left(\frac{t}{\alpha}\right)^{\beta}}$, where the shape parameter $\beta$ and the scale parameter $\alpha$ are randomly selected from the uniform distribution on the intervals $(0.5,4)$ and $(12,60)$, respectively.

(3) If a component fails, a new identical component with the same $\alpha$ and $\beta$ as the failed one replaces the failed component.

(4) In each case we use 10 replicates of each system so that $M=10$. 
(5) We use the values 15, 20 for $M_{j}$, the number of failures observed for each replicate.

(6) Using the randomly generated data implied by the above, we fit each of the 6 models: the RP, the GP, the GRP, the NHPP-PL, Model I, and Model II, by maximising their respective log-likelihoods. For Model I and Model II the likelihoods are given by Eqs (11) and (12). The reader is referred to Lam (2007) for the likelihoods of the RP, the GP and the NHPP-PL, respectively, and to Yanez, Joglar, and Modarres (2002) for the likelihood of the GRP. In the RP, the GP, and the GRP, it is assumed that time to first failure is a Weibull distribution $1-e^{-\left(\frac{t}{\alpha_{1}}\right)^{\beta_{1}}}$. In the NHPP-PL model, $\left(\frac{t}{\alpha_{1}}\right)^{\beta_{1}-1}$ is the CRIF of the series system. In both Model I and Model II, we assume that $h_{\mathrm{c}}(t)=\frac{\beta_{1}}{\alpha_{1}}\left(\frac{t}{\alpha_{1}}\right)^{\beta_{1}-1}$ and $\lambda_{\mathrm{s}}(t)=\frac{\beta_{2}}{\alpha_{2}}\left(\frac{t}{\alpha_{2}}\right)^{\beta_{2}-1}$.

(7) Then we calculate the AIC (Akaike information criterion) value for each model:

$$
\mathrm{AIC}=2 p-2 \ln (L)
$$

where $L$ is the maximized value of the log-likelihood for the model, $p$ is the number of parameters in the model. The term $2 p$ in the AIC penalises a model with a large number of parameters. The values of $p$ of the six models are shown in Table 1. Note, Model I and Model II with $p=4$ incur the highest penalty on their AIC values.

Table 1

The number of parameters $p$ for each model.

\begin{tabular}{c|cccccc}
\hline & RP & GP & NHPP-PL & GRP & Model I & Model II \\
\hline$p$ & 2 & 3 & 2 & 3 & 4 & 4 \\
\hline
\end{tabular}

With $M_{j}=15$ and $M_{j}=20$ and $m=5,10,15$ there are 6 combinations. We repeat the above steps (1) to (7) for each of 30 repetitions, in each of which the values of $\alpha$ and $\beta$ are different. Calculations are done with a statistical package $\mathrm{R}$ (which can be downloaded from www.rproject.org/). Table 2 shows the mean values and the variances (that are shown in the brackets under means) of the AIC values of the models over the 30 repetition for each combination.

On the 30 repetitions of each of the 6 combinations, we then use a paired t-test at the 5 percent level to compare the AIC values of Model I and Model II with each of the other four models, respectively. The results are shown in Table 3, which includes 48 comparisons in total. In the table, symbol ' $\checkmark$ ' represents the result that the hypothesis test fails to reject the null hypothesis that the AIC value of Model I (or Model II) is smaller than that of its comparison model, and symbol ' $x$ ' represents the result that the hypothesis test rejects the null hypothesis. From the table, we make the following observations.

- On all the comparisons, both Model I and Model II have smaller AIC values than those of 
the RP and the GP.

- On the performance of Model I. In only 8 out of 24 comparisons does the hypothesis test reject the null hypothesis that the AIC value of Model I is smaller than that of the NHPP-PL or that of the GRP. Thus, in the remaining 16 of the 24 comparisons the test fails to reject the null hypothesis.

- On the performance of Model II. In all the comparisons the test fails to reject the null hypothesis. That is, in all the comparisons, Model II outperforms the other models.

- Comparing the AIC values of Model I and Model II, it can be seen that Model II outperforms Model I.

Table 2

The mean values of AIC from 30 repetitions, for various values of the number of replicates, $M$, number of failures $M_{j}$ of each replicate, and number of components $m$ in each replicate of the system.

\begin{tabular}{|c|c|c|c|c|c|c|c|c|}
\hline \multirow[b]{2}{*}{$M$} & \multirow[b]{2}{*}{$M_{j}$} & \multirow[b]{2}{*}{$m$} & \multicolumn{6}{|c|}{ AIC values } \\
\hline & & & $\mathrm{RP}$ & GP & NHPP-PL & GRP & Model I & Model II \\
\hline \multirow{6}{*}{10} & \multirow{3}{*}{15} & $\underline{5}$ & $\begin{array}{l}855.73 \\
(77.25)\end{array}$ & $\begin{array}{l}856.42 \\
(74.92)\end{array}$ & $\begin{array}{l}856.48 \\
(73.45)\end{array}$ & $\begin{array}{l}853.53 \\
(75.20)\end{array}$ & $\begin{array}{l}851.81 \\
(75.33)\end{array}$ & $\begin{array}{l}\underline{840.95} \\
(74.83)\end{array}$ \\
\hline & & 10 & $\begin{array}{l}670.60 \\
(50.69)\end{array}$ & $\begin{array}{l}672.39 \\
(50.68)\end{array}$ & $\begin{array}{l}663.45 \\
(48.25)\end{array}$ & $\begin{array}{l}663.22 \\
(48.13)\end{array}$ & $\begin{array}{l}662.98 \\
(47.91)\end{array}$ & $\begin{array}{l}\underline{659.31} \\
(47.88)\end{array}$ \\
\hline & & 15 & $\begin{array}{l}562.87 \\
(36.72)\end{array}$ & $\begin{array}{l}564.77 \\
(36.79)\end{array}$ & $\begin{array}{l}553.70 \\
(34.74)\end{array}$ & $\begin{array}{l}554.04 \\
(35.33)\end{array}$ & $\begin{array}{l}553.75 \\
(35.18)\end{array}$ & $\begin{array}{l}\underline{551.34} \\
(34.96)\end{array}$ \\
\hline & \multirow{3}{*}{20} & 5 & $\begin{array}{l}1126.03 \\
(107.52)\end{array}$ & $\begin{array}{l}1125.65 \\
(103.85)\end{array}$ & $\begin{array}{l}1125.50 \\
(102.58)\end{array}$ & $\begin{array}{l}1121.52 \\
(105.03)\end{array}$ & $\begin{array}{l}1119.72 \\
(104.70)\end{array}$ & $\frac{1109.49}{(105.42)}$ \\
\hline & & 10 & $\begin{array}{l}877.23 \\
(68.15) \\
\end{array}$ & $\begin{array}{l}877.36 \\
(65.43)\end{array}$ & $\begin{array}{l}868.31 \\
(64.89)\end{array}$ & $\begin{array}{l}867.82 \\
(64.72) \\
\end{array}$ & $\begin{array}{l}867.69 \\
(64.32) \\
\end{array}$ & $\begin{array}{l}\underline{862.78} \\
(65.03)\end{array}$ \\
\hline & & 15 & $\begin{array}{l}711.76 \\
(57.32)\end{array}$ & $\begin{array}{l}711.08 \\
(58.90)\end{array}$ & $\begin{array}{l}699.44 \\
(57.95)\end{array}$ & $\begin{array}{c}700.16 \\
(57.82)\end{array}$ & $\begin{array}{l}700.09 \\
(58.04)\end{array}$ & $\begin{array}{l}\underline{695.76} \\
(57.09)\end{array}$ \\
\hline
\end{tabular}

To consider further the performance of Model I and Model II, we investigate the case $M=10$, $M_{j}=15$, and $m=5$ in more detail. The results are shown in Table 4 . As can be seen from this table, the AIC values of the GRP are smaller than those of the NHPP-PL on most repetitions (precisely, on 23 repetitions). We therefore compare the AIC values of Model I and Model II with the AIC of the GRP. Based on Table 4, we plot Figure 4 that shows the differences between the AIC values of the GRP and those of Model I (i.e., the solid line), and the differences between the AIC values of the GRP and Model II (i.e., the dashed line) on the 30 repetitions. From the figure, we make the following observations 
Table 3

Comparison of the AIC values of the six models based on the paired t-tests (at 5 percent level).

\begin{tabular}{|c|c|c|c|c|c|c|c|c|c|c|}
\hline & \multicolumn{4}{|c|}{ Is Model I's AIC value smaller than that of } & \multicolumn{4}{|c|}{ Is Model II's AIC value smaller than that of } \\
\hline$M$ & $M_{j}$ & $m$ & $\mathrm{RP} ?$ & GP? & NHPP-PL? & GRP? & $\mathrm{RP} ?$ & GP? & NHPP-PL? & GRP? \\
\hline \multirow{6}{*}{10} & \multirow{3}{*}{15} & 5 & $\checkmark$ & $\checkmark$ & $\checkmark$ & $\checkmark$ & $\checkmark$ & $\checkmark$ & $\checkmark$ & $\checkmark$ \\
\hline & & 10 & $\checkmark$ & $\checkmark$ & $x$ & $x$ & $\checkmark$ & $\checkmark$ & $\checkmark$ & $\checkmark$ \\
\hline & & 15 & $\checkmark$ & $\checkmark$ & $\times$ & $\times$ & $\checkmark$ & $\checkmark$ & $\checkmark$ & $\checkmark$ \\
\hline & \multirow{3}{*}{20} & 5 & $\checkmark$ & $\checkmark$ & $\checkmark$ & $\checkmark$ & $\checkmark$ & $\checkmark$ & $\checkmark$ & $\checkmark$ \\
\hline & & 10 & $\checkmark$ & $\checkmark$ & $\times$ & $\times$ & $\checkmark$ & $\checkmark$ & $\checkmark$ & $\checkmark$ \\
\hline & & 15 & $\checkmark$ & $\checkmark$ & $x$ & $x$ & $\checkmark$ & $\checkmark$ & $\checkmark$ & $\checkmark$ \\
\hline
\end{tabular}

- All the AIC differences between the GRP and Model II are positive. Thus Model II performs better than the GRP on all 30 repetitions. The largest two AIC differences are greater than 24 (i.e, the values corresponding 9 and 30 on the $\mathrm{X}$-axis).

- When the AIC differences between the GRP and Model I are compared, on 10 of 30 repetitions the AIC values of the GRP are greater than that of Model I. The largest AIC difference is greater than 15 (i.e, the value corresponding 9 on the $\mathrm{X}$-axis).

Furthermore, to allow the reader who may be interested to validate the results by repeating the experiment, we record model parameters generated in one of the 30 repetitions from the case in which $M_{j}=15$ and $m=5$. Table 5 shows the Weibull distribution parameters used to generate the time between failures in this repetition. With these parameters, the random lifetimes of the components in a system (here $M=1$ ) are generated (Table 6). Table 7 shows the estimated parameters and AIC for the six models. It can be seen that (1) in Model I, $\beta_{2}$ is smaller than 1, which implies that the hazard function $\lambda_{s}(t)$ of VC2 in Model I decreases over time, (2) the AIC values of Model I and Model II are the smallest among the six models, and (3) the values of $\alpha_{1}$ and $\beta_{1}$ in the RP and the GP are the same and the values of $\alpha_{1}$ and $\beta_{1}$ in the NHPP-PL and the GRP are the same. Hence, the GP and the GRP will not be further analysed. Figure 5 shows the CIFs of the other four models and the empirical CIF (i.e., the cumulative number of failures) generated from the data shown in Table 6. From the figure, we can see that Model I and Model II fit the empirical CIF well and the RP has the worst fit, which confirms its large AIC value shown in Table 7. 
Table 4

Estimated AIC values of the six models for each individual repetition for the case when $M=10$, $M_{j}=15$ and $m=5$, corresponding to the first row (with the $m$ value underlined) in Table 2.

\begin{tabular}{|c|c|c|c|c|c|c|c|c|c|c|c|c|c|}
\hline repetition & $\mathrm{RP}$ & GP & NHPP-PL & GRP & Model I & Model II & repetition & $\mathrm{RP}$ & GP & NHPP-PL & GRP & Model I & Model II \\
\hline 1 & 779.35 & 779.77 & 779.30 & 775.36 & 771.82 & 765.41 & 16 & 979.41 & 981.35 & 974.07 & 972.86 & 978.07 & 961.13 \\
\hline 2 & 760.67 & 762.01 & 762.60 & 760.15 & 757.16 & 743.85 & 17 & 865.21 & 866.93 & 866.02 & 863.35 & 864.37 & 849.03 \\
\hline 3 & 927.23 & 928.67 & 925.81 & 924.50 & 923.12 & 917.20 & 18 & 947.38 & 949.31 & 946.13 & 946.58 & 948.49 & 934.47 \\
\hline 4 & 818.35 & 820.30 & 820.40 & 819.94 & 816.98 & 800.98 & 19 & 750.29 & 752.26 & 750.74 & 748.28 & 748.41 & 732.82 \\
\hline 5 & 888.91 & 890.05 & 891.50 & 885.54 & 887.90 & 880.61 & 20 & 845.51 & 847.50 & 854.76 & 844.79 & 838.28 & 833.61 \\
\hline 6 & 781.63 & 783.36 & 800.80 & 783.49 & 783.01 & 773.31 & 21 & 911.38 & 912.37 & 915.41 & 910.11 & 909.89 & 893.32 \\
\hline 7 & 921.32 & 922.94 & 914.74 & 916.74 & 914.38 & 906.13 & 22 & 845.37 & 846.10 & 842.61 & 841.98 & 835.94 & 829.25 \\
\hline 8 & 829.80 & 830.55 & 834.07 & 826.89 & 820.26 & 808.77 & 23 & 697.75 & 698.90 & 695.11 & 694.05 & 693.98 & 684.63 \\
\hline 9 & 1004.75 & 982.86 & 965.87 & 976.12 & 960.30 & 944.94 & 24 & 808.40 & 810.02 & 813.92 & 809.28 & 803.59 & 801.11 \\
\hline 10 & 866.31 & 866.35 & 873.63 & 866.88 & 862.58 & 858.14 & 25 & 867.02 & 868.87 & 870.00 & 866.54 & 862.72 & 847.86 \\
\hline 11 & 788.51 & 789.78 & 790.82 & 788.76 & 790.61 & 772.71 & 26 & 923.48 & 925.06 & 922.86 & 924.50 & 926.69 & 922.81 \\
\hline 12 & 767.24 & 768.95 & 776.46 & 769.24 & 770.09 & 766.81 & 27 & 729.82 & 730.96 & 729.32 & 727.85 & 725.40 & 716.88 \\
\hline 13 & 902.65 & 904.55 & 911.43 & 903.90 & 903.53 & 902.94 & 28 & 954.91 & 956.90 & 954.95 & 956.78 & 958.16 & 943.80 \\
\hline 14 & 936.27 & 938.12 & 929.67 & 930.86 & 929.84 & 917.80 & 29 & 881.00 & 883.00 & 881.11 & 878.75 & 876.43 & 861.49 \\
\hline 15 & 839.34 & 841.20 & 840.10 & 841.34 & 841.82 & 832.72 & 30 & 852.60 & 853.68 & 860.35 & 850.59 & 850.53 & 823.91 \\
\hline
\end{tabular}

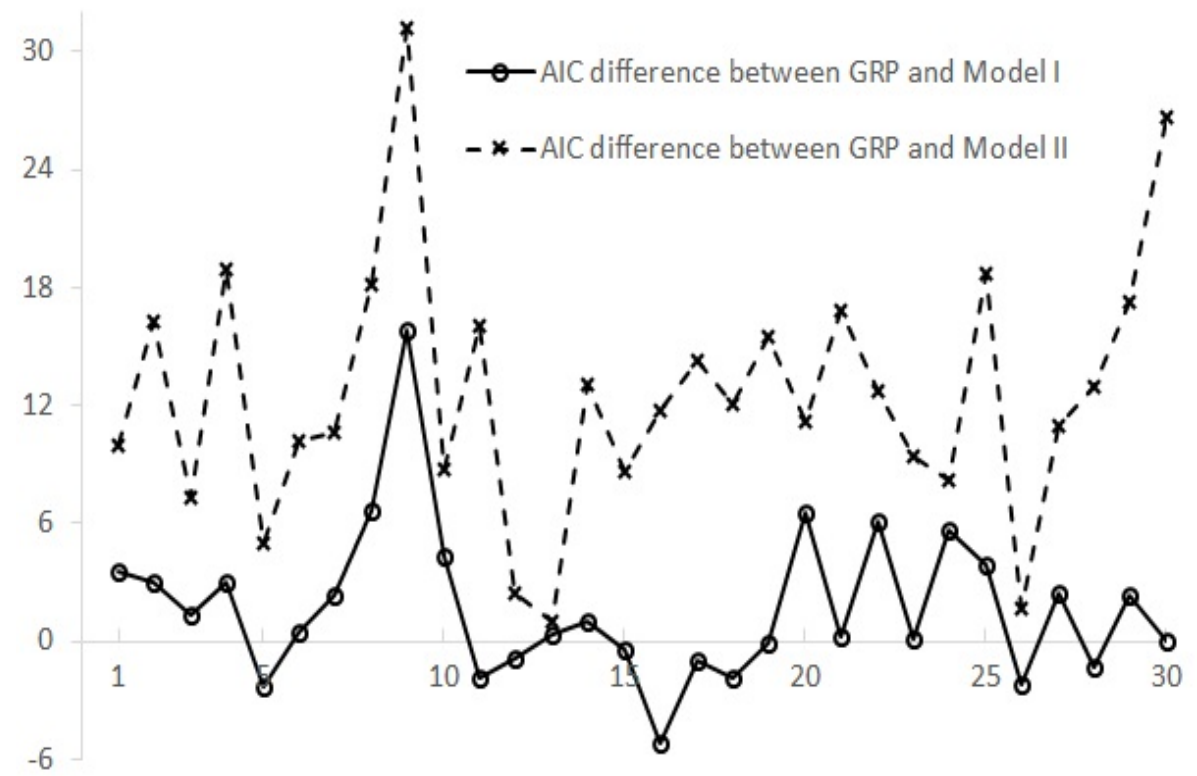

Fig. 4. The difference in the AIC values between Model I and GRP, and the difference in the AIC values between Model II and GRP, based on the data shown in Table 4. 
Table 5

The parameters of the Weibull distributions of the 5 components used to generate failures in e the case $M_{j}=15$ and $m=5$.

\begin{tabular}{cc|cc|cc|cc|cc}
\hline$\alpha$ & $\beta$ & $\alpha$ & $\beta$ & $\alpha$ & $\beta$ & $\alpha$ & $\beta$ & $\alpha$ & $\beta$ \\
\hline 26.725 & 2.276 & 53.900 & 2.893 & 48.890 & 3.571 & 33.008 & 2.981 & 35.435 & 0.846 \\
\hline
\end{tabular}

Table 6

15 observations generated using the parameters in Table 5 .

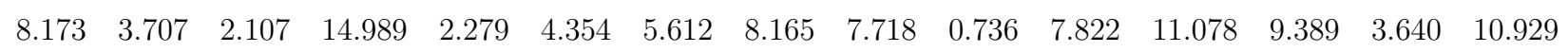

Table 7

Results for the models fitted to the simulated times between failures given in Table 6 .

\begin{tabular}{c|c|c|c|c|c}
\hline \multicolumn{5}{|c}{ Estimated Parameters } \\
\hline RP & GP & NHPP-PL & GRP & Model I & Model II \\
\hline$\hat{\alpha}=26.286$ & $\hat{a}=1.000$ & $\hat{\alpha}=8.374$ & $\hat{q}=1.0001$ & $\hat{\alpha_{1}}=10.105, \hat{\beta_{1}}=2.764$ & $\hat{\alpha_{1}}=11.072, \hat{\beta}_{1}=3.144$ \\
$\hat{\beta}=0.829$ & $\hat{\alpha}=26.286, \hat{\beta}=0.829$ & $\hat{\beta}=1.0889$ & $\hat{\alpha}=8.374, \hat{\beta}=1.0889$ & $\hat{\alpha_{2}}=15.213, \hat{\beta_{2}}=0.961$ & $\hat{\alpha}_{2}=20.429, \hat{\beta_{2}}=1.905$ \\
$\mathrm{AIC}=108.941$ & $\mathrm{AIC}=110.941$ & $\mathrm{AIC}=91.018$ & $\mathrm{AIC}=93.017$ & $\mathrm{AIC}=89.093$ & $\mathrm{AIC}=88.161$ \\
\hline
\end{tabular}

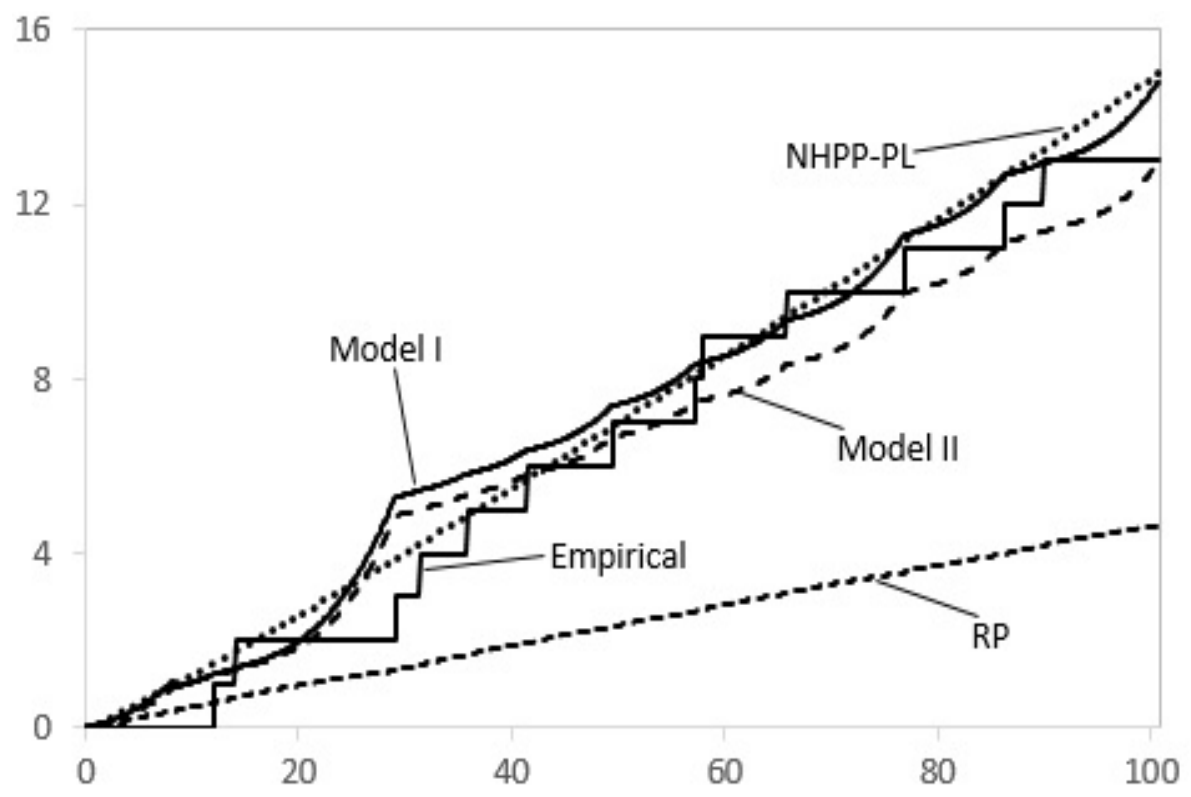

Fig. 5. The cumulative number of failures (empirical) and the CIFs for the models fitted to the simulated times between failures given in Table 6 .

\subsection{A real dataset}

In this subsection we compare the models when fitted to a dataset (TBF-7913) published in Proschan (1963). The data are the 27 observations of times-between-failure of an air conditioning unit in an aircraft.

Since the number of components in the system is not specified, following Remark 3, we simply 
compare the AIC values of Model II when the number of components is assumed to be 2, 3, ..., and 10. The minimum AIC value is achieved when the number of components is 3 . The parameters and the AIC values of the models are listed in Table 8, from which we can see that Model II outperforms other models.

Table 8

Results for the models fitted to the air conditioner data TBF-7913. Estimated Parameters

\begin{tabular}{c|c|c|c|c|c}
\hline RP & GP & NHPP-PL & GRP & Model I & Model II \\
\hline$\hat{\alpha}=79.924$ & $\hat{a}=0.971$ & $\hat{\alpha}=73.739$ & $\hat{q}=0.0000001$ & $\hat{\alpha_{1}}=219.978, \hat{\beta}_{1}=2.988$ & $\hat{\alpha_{1}}=176.503, \hat{\beta_{1}}=1.897$ \\
$\hat{\beta}=1.123$ & $\hat{\alpha}=54.917, \hat{\beta}=1.148$ & $\hat{\beta}=0.988$ & $\hat{\alpha}=49.438, \hat{\beta}=1.123$ & $\hat{\alpha_{2}}=93.558, \hat{\beta}_{2}=1.00855$ & $\hat{\alpha_{2}}=77.406, \hat{\beta_{2}}=0.875$ \\
$\mathrm{AIC}=293.913$ & $\mathrm{AIC}=292.202$ & $\mathrm{AIC}=292.431$ & $\mathrm{AIC}=293.913$ & $\mathrm{AIC}=294.578$ & $\mathrm{AIC}=\mathbf{2 9 0 . 8 4 2}$ \\
\hline
\end{tabular}

\section{Conclusions}

This paper develops two stochastic models of the failure process of a multi-component series system. Model I regards the failure process of the system equivalent to that of a virtual system consisting of two sockets into each of which there is inserted a virtual component. Whenever the system fails, replacement occurs at socket 1 and minimal repair or no maintenance is conducted on the virtual component in socket 2. Model II regards the failure process of the system equivalent to that of a virtual system consisting of a socket and a subsystem. The socket contains one virtual component and the subsystem contains $m-1$ sockets into each of which there is inserted virtual components. Broadly speaking, whenever the virtual system fails, replacement occurs at socket 1 and the virtual subsystem is imperfectly repaired.

The performance of the two models is compared with four well-known models on the basis of six artificially generated datasets. The results show that (1) overall Model I outperforms the renewal process and the geometric process on the simulated data and (2) Model II outperforms the four models on the simulated data and on the real data. Model II performs better than Model I. Furthermore, when fitted to a real-world dataset, Model II is again the best performing model of the six.

The models quantify the notion that the repair effect must be at least as big as if the most reliable component were replaced, and therefore the models consider a repair effect through component replacement rather than through the parameterization of maintenance effectiveness.

In a further applied study, we intend to fit the models to a large number of real datasets, and handle the case of right censoring in the likelihood estimation of the models. Finally, we make the point that methods that do not make parametric assumptions about the hazard function $h_{c}(x)$ and the intensity function $\lambda_{s}(x)$ are appealing in many settings, and our future work may consider the development of non-parametric versions of Models I and Model II. 


\section{Acknowledgements}

The authors are indebted to the reviewers and the editor for their constructive comments.

\section{References}

Andersen, P. K., Borgan, O., Gill, R. D., \& Keiding, N. (1993). Statistical models based on counting processes. New York: Springer Science \& Business Media.

Ascher, H., \& Feingold, H. (1984). Repairable Systems Reliability Modeling, Inference, Misconceptions and Their Causes. New York: Marcel-Dekker.

Asfaw, Z. G., \& Lindqvist, B. H. (2015). Extending minimal repair models for repairable systems: A comparison of dynamic and heterogeneous extensions of a nonhomogeneous poisson process. Reliability Engineering \& System Safety, 140, 53-58.

Baxter, L. A., Kijima, M., \& Tortorella, M. (1996). A point process model for the reliability of a maintained system subject to general repair. Stochastic Models, 12(1), 12-1.

Cox, D., \& Lewis, P. (1966). The statistical analysis of series of events. London: John Wiley and Sons.

Dorado, C., Hollander, M., \& Sethuraman, J. (1997). Nonparametric estimation for a general repair model. The Annals of Statistics, 25(3), 1140-1160.

Doyen, L., \& Gaudoin, O. (2004). Classes of imperfect repair models based on reduction of failure intensity or virtual age. Reliability Engineering $\&$ System Safety, 84(1), 45-56.

Doyen, L., \& Gaudoin, O. (2011). Modeling and assessment of aging and efficiency of corrective and planned preventive maintenance. IEEE Transactions on Reliability, 60 (4), 759-769.

Drenick, R. F. (1960). The failure law of complex equipment. Journal of the Society for Industrial \& Applied Mathematics, 8(4), 680-690.

Duane, J. (1964). Learning curve approach to reliability monitoring. IEEE Transactions on Aerospace, 2(2), 563-566.

Gilardoni, G. L., Toledo, M. L. G. de, Freitas, M. A., \& Colosimo, E. A. (2015). Dynamics of an optimal maintenance policy for imperfect repair models. European Journal of Operational Research.

Høyland, A., \& Rausand, M. (2009). System reliability theory: Models and statistical methods (Vol. 420). New Jersey: John Wiley \& Sons.

Kijima, M. (1989). Some results for repairable systems with general repair. Journal of Applied Probability, 26(1), 89-102.

Kijima, M., \& Sumita, U. (1986). A useful generalization of renewal theory: counting processes 
governed by non-negative markovian increments. Journal of Applied Probability, 23(1), 71-88.

Lam, Y. (1988). Geometric processes and replacement problem. Acta Mathematicae Applicatae Sinica, 4, 366-377.

Lam, Y. (2007). The geometric process and its applications. Singapore: World Scientific.

Meeker, W. Q., \& Escobar, L. A. (1998). Statistical methods for reliability data. New York: John Wiley \& Sons.

Proschan, F. (1963). Theoretical explanation of observed decreasing failure rate. Technometrics, $5(3), 375-383$.

Ross, S. M. (1996). Stochastic processes (2nd ed.). New York: John Wiley \& Sons.

Wang, H., \& Pham, H. (1996). A quasi renewal process and its applications in imperfect maintenance. International Journal of Systems Science, 27(10), 1055-1062.

Wu, S., \& Clements-Croome, D. (2006). A novel repair model for imperfect maintenance. IMA Journal Management Mathematics, 17(3), 235-243.

Wu, S., \& Scarf, P. (2015). Decline and repair, and covariate effects. European Journal of Operational Research, 244(1), 219-226.

Wu, S., \& Zuo, M. (2010). Linear and nonlinear preventive maintenance models. IEEE Transactions on Reliability, 59(1), 242-249.

Yanez, M., Joglar, F., \& Modarres, M. (2002). Generalized renewal process for analysis of repairable systems with limited failure experience. Reliability Engineering 83 System Safety, r7 (2), 167-180.

Zhang, M., Gaudoin, O., \& Xie, M. (2015). Degradation-based maintenance decision using stochastic filtering for systems under imperfect maintenance. European Journal of Operational Research, $245(2), 531-541$. 
governed by non-negative markovian increments. Journal of Applied Probability, 23(1), 71-88.

Lam, Y. (1988). Geometric processes and replacement problem. Acta Mathematicae Applicatae Sinica, 4, 366-377.

Lam, Y. (2007). The geometric process and its applications. Singapore: World Scientific.

Meeker, W. Q., \& Escobar, L. A. (1998). Statistical methods for reliability data. New York: John Wiley \& Sons.

Proschan, F. (1963). Theoretical explanation of observed decreasing failure rate. Technometrics, $5(3), 375-383$.

Ross, S. M. (1996). Stochastic processes (2nd ed.). New York: John Wiley \& Sons.

Wang, H., \& Pham, H. (1996). A quasi renewal process and its applications in imperfect maintenance. International Journal of Systems Science, 27(10), 1055-1062.

Wu, S., \& Clements-Croome, D. (2006). A novel repair model for imperfect maintenance. IMA Journal Management Mathematics, 17(3), 235-243.

Wu, S., \& Scarf, P. (2015). Decline and repair, and covariate effects. European Journal of Operational Research, 244(1), 219-226.

Wu, S., \& Zuo, M. (2010). Linear and nonlinear preventive maintenance models. IEEE Transactions on Reliability, 59(1), 242-249.

Yanez, M., Joglar, F., \& Modarres, M. (2002). Generalized renewal process for analysis of repairable systems with limited failure experience. Reliability Engineering 83 System Safety, r7 (2), 167-180.

Zhang, M., Gaudoin, O., \& Xie, M. (2015). Degradation-based maintenance decision using stochastic filtering for systems under imperfect maintenance. European Journal of Operational Research, $245(2), 531-541$. 